\title{
A method of calculation for the determinant of the Potts model transfer matrix
}

\author{
B. Mirza and M.R. Bakhtiari \\ Department of Physics, Isfahan University of Technology, Isfahan 84154, Iran \\ E-mail: b.mirza@cc.iut.ac.ir
}

\begin{abstract}
By using a decomposition of the transfer matrix of the two dimensional $q$-state Potts Model to $V_{1}^{\prime}$ and $V_{2}$ its determinant is calculated. Our result is a proof for a conjectured formula by Chang and Shrock in [14].
\end{abstract}

PACS numbers: 05.50.+q, 02.70.-c

Keywords: Potts Model; Exactly Solvable Models. 


\section{Introduction}

The two dimensional $q$-state Potts models $[1,2]$ for various $q$ have been of interest as examples of different universality classes for phase transitions and, for $q=3,4$ as models for the adsorption of gases on certain substrates [3,4,5]. For $q \geq 3$ the free energy has never been calculated in closed form for arbitrary temperature. It is thus of continuing value to obtain further information about the two dimensional Potts model. Some exact results have been established for the model: from a duality relation, the critical point has been identified [1]. The free energy and latent heat [6,7,8], and magnetization [9] have been calculated exactly by Baxter at this critical point, establishing that the model has a continuous, second order transition for $q \leq 4$ and a first order transition for $q \geq 5$. Baxter has also shown that although the $q=3$ model has no phase with antiferromagnetic long-range order at any finite temperature there is an antiferromagnetic critical point at $T=0$ [9]. The values of the critical exponents (for the range of $q$ where the transition is continuous) have been determined [10,11,12]. Further insight into the critical behaviour was gained using the methods of conformal field theory [13]. In this paper a proof for a conjectured formula by Chang and Shrock [14] for determinants of the transfer matrices of the $q$-state Potts model is given. The paper is organized as follows: In section II, by using the standard representation for the transfer matrices of the $q$-state Potts model [15] the determinants of the transfer matrices for $n \times n$ lattices with periodic boundary conditions are calculated.

\section{Determinant of the Potts model transfer matrix}

The $q$-state Potts model has served as a valuable model in the study of phase transition and critical phenomena. On a lattice, or more generally on a graph $G$, at temperature $T$ this model is defined by the partition function:

$$
Z(G, q, k)=\sum_{\left\{\sigma_{n}\right\}} e^{-\beta H}
$$

with the Hamiltonian

$$
H=-J \sum_{<i, j>} \delta\left(s_{i}, s_{j}\right)
$$

where $\delta\left(s_{i}, s_{j}\right)$ is the kronecker delta and $s_{i}=1, \ldots, q$ are the spin variables on each vertex $i \in G, \beta=\left(k_{B} T\right)^{-1}, k=\beta J$; and $\langle i, j\rangle$ denotes pairs of adjacent vertices. Consider an $n \times n$ square lattice with periodic boundary conditions. For the Ising model, partition function can be written as product of transfer matrices and the eigenvalues can be calculated exactly $[15,16,17,18,19,20]$. There are also several representations for the transfer matrix of the $q$-state Potts model $[15,21]$. Following the method which is used in $[15,16,17,21]$ for representation of the transfer matrices of the two dimensional Ising model and the $q$-state Potts model, we use a decomposition of the transfer matrices which helps us to obtain the determinant of the matrices.

Consider a square lattice of $N=n^{2}$ spins consisting of $n$ rows and $n$ columns with a toroidal boundary condition. Let $\gamma_{\alpha} \equiv\left\{s_{1}, s_{2}, \ldots, s_{n}\right\}$ denote the collection of all spin 
coordinates of the $\alpha$ th row $(\alpha=1, \ldots, n)$ with a toroidal boundary condition $\gamma_{n+1} \equiv \gamma_{1}$. A configuration of the entire lattice is then specified by $\left\{\gamma_{1}, \ldots, \gamma_{n}\right\}$. Let $E\left(\gamma_{\alpha}, \gamma_{\alpha+1}\right)$ be the interaction energy between the $\alpha$ th and the $(\alpha+1)$ th row and $E\left(\gamma_{\alpha}\right)$ be the interaction energy of spins within $\alpha$ th row. We can write

$$
\begin{aligned}
& E\left(\gamma, \gamma^{\prime}\right)=-J \sum_{k=1}^{n} \delta\left(s_{k}, s_{k}^{\prime}\right) \\
& E(\gamma)=-J \sum_{k=1}^{n} \delta\left(s_{k}, s_{k+1}\right)
\end{aligned}
$$

where $\gamma \equiv\left\{s_{1}, \ldots, s_{n}\right\}$ and $\gamma^{\prime} \equiv\left\{s_{1}^{\prime}, \ldots, s_{n}^{\prime}\right\}$ respectively denote the collection of spin coordinates in two neighboring rows and the partition function is

$$
Z_{P F}=\sum_{\gamma_{1}} \ldots \sum_{\gamma_{n}} \exp \left\{-\beta \sum_{\alpha=1}^{n}\left[E\left(\gamma_{\alpha}, \gamma_{\alpha+1}\right)+E\left(\gamma_{\alpha}\right)\right]\right\}
$$

Let a $q^{n} \times q^{n}$ matrix $P$ be so defined that its matrix elements are

$$
<\gamma|P| \gamma^{\prime}>\equiv e^{-\beta\left[E\left(\gamma, \gamma^{\prime}\right)+E(\gamma)\right]}
$$

Then

$$
Z_{P F}=\sum_{\gamma_{1}} \ldots \sum_{\gamma_{n}}<\gamma_{1}|P| \gamma_{2}><\gamma_{2}|P| \gamma_{3}>\ldots<\gamma_{n}|P| \gamma_{1}>=\operatorname{Tr} P^{n}
$$

From $(3),(4)$ and (6) we may obtain the matrix elements of $P$ in the form

$$
<s_{1}, \ldots, s_{n}|P| s_{1}^{\prime}, \ldots, s_{n}^{\prime}>=\prod_{k=1}^{n} e^{k \delta\left(s_{k}, s_{k+1}\right)} e^{k \delta\left(s_{k}, s_{k}^{\prime}\right)}
$$

Let us define two $q^{n} \times q^{n}$ matrices $V_{1}^{\prime}$ and $V_{2}$ whose matrix elements are given by [15]

$$
\begin{gathered}
<s_{1}, \ldots, s_{n}\left|V_{1}^{\prime}\right| s_{1}^{\prime}, \ldots, s_{n}^{\prime}>\equiv \prod_{k=1}^{n} e^{k \delta\left(s_{k}, s_{k}^{\prime}\right)} \\
<s_{1}, \ldots, s_{n}\left|V_{2}\right| s_{1}^{\prime}, \ldots, s_{n}^{\prime}>\equiv \delta\left(s_{1}, s_{1}^{\prime}\right) \ldots \delta\left(s_{n}, s_{n}^{\prime}\right) \prod_{k=1}^{n} e^{k \delta\left(s_{k}, s_{k+1}\right)}
\end{gathered}
$$

where $V_{2}$ is a diagonal matrix in the present representation. It is easily verified that $P=V_{2} V_{1}^{\prime}$, or it can be written as

$$
\begin{gathered}
<s_{1}, \ldots, s_{n}|P| s_{1}^{\prime}, \ldots, s_{n}^{\prime}>= \\
\sum_{s_{1}^{\prime \prime}, \ldots, s_{n}^{\prime \prime}}<s_{1}, \ldots, s_{n}\left|V_{2}\right| s_{1}^{\prime \prime}, \ldots, s_{n}^{\prime \prime}><s_{1}^{\prime \prime}, \ldots, s_{n}^{\prime \prime}\left|V_{1}^{\prime}\right| s_{1}^{\prime}, \ldots, s_{n}^{\prime}>
\end{gathered}
$$


Let $A_{1}$ and $A_{2}$ be two $m \times m$ matrices whose elements are respectively $\left\langle i\left|A_{1}\right| j\right\rangle$ and $<i\left|A_{2}\right| j>$, where $i$ and $j$ independently take on the values $1,2, \ldots, m$. Then the direct product $A_{1} \otimes A_{2}$ is the $m^{2} \times m^{2}$ matrix whose matrix elements are

$$
<i_{1} i_{2}\left|A_{1} \otimes A_{2}\right| j_{1} j_{2}>=<i_{1}\left|A_{1}\right| j_{1}><i_{2}\left|A_{1}\right| j_{2}>
$$

This definition can be immediately extended to define the direct product $A_{1} \otimes A_{2} \otimes \cdots \otimes A_{n}$ of any number of $m \times m$ matrices $A_{1}, A_{2}, \ldots, A_{n}$ :

$$
\begin{aligned}
& <i_{1} i_{2} \cdots i_{n}\left|A_{1} \otimes A_{2} \otimes \cdots \otimes A_{n}\right| j_{1} j_{2} \cdots j_{n}>= \\
& <i_{1}\left|A_{1}\right| j_{1}><i_{2}\left|A_{2}\right| j_{2}>\cdots<i_{n}\left|A_{n}\right| j_{n}>
\end{aligned}
$$

By inspection of (9) it is clear that $V_{1}^{\prime}$ is a product of $n q \times q$ identical matrices

$$
V_{1}^{\prime}=A \otimes A \otimes \cdots \otimes A
$$

where

$$
<s|A| s^{\prime}>=e^{k \delta\left(s, s^{\prime}\right)}
$$

Therefore

$$
A=\left(\begin{array}{cccc}
e^{k} & 1 & \ldots & 1 \\
1 & e^{k} & & \\
\vdots & & \ddots & \\
1 & \ldots & & e^{k}
\end{array}\right)=e^{k} I_{q \times q}+\sigma_{q \times q}
$$

where $\sigma$ is a $q \times q$ matrix with zero diagonal elements and unit elements on all other entries (note that $\sigma^{2}=(q-2) \sigma+(q-1) I$ ) and $I$ is a $q \times q$ unit matrix. Let us represent $A$ by the following equation

$$
A=f(k) e^{\frac{\tilde{\kappa}}{2} X} \quad, \quad X^{2} \equiv I_{q \times q}
$$

where $f$ is a function of $k$ and a condition is imposed on $X . \tilde{k}$ is the dual of $k$ which is given by the duality relation

$$
e^{-\tilde{k}}=\frac{e^{k}-1}{e^{k}+(q-1)}
$$

By considering a linear relation between $X$ and $\sigma\left(X_{q \times q}=a \sigma_{q \times q}+b I_{q \times q}\right)$ we can calculate $f(k)$ and $X$. After a straightforward calculation we arrive at

$$
\begin{aligned}
& X=\frac{2}{q} \sigma+\left(\frac{2}{q}-1\right) I \\
& f(k)=\left(e^{k}-1\right) e^{\frac{\tilde{\underline{x}}}{2}}
\end{aligned}
$$


Hence

$$
\begin{aligned}
V_{1}^{\prime} & =\left[\left(e^{k}-1\right) e^{\frac{\tilde{k}}{2}}\right]^{n} \exp \left(\frac{\tilde{k}}{2} \sum_{\alpha=1}^{n} X_{\alpha}\right) \\
& =\left[\left(e^{k}-1\right) e^{\frac{\tilde{k}}{2}}\right]^{n} V_{1} \\
X_{\alpha} & =1 \otimes \ldots \otimes 1 \otimes X \otimes 1 \otimes \ldots \otimes 1
\end{aligned}
$$

where $X$ is the $\alpha$ th factor. In this part we will use the following representation for $V_{2}$ which is a result of its definition in (10).

$$
\begin{gathered}
V_{2}=\prod_{\alpha=1}^{n} e^{\left(\frac{k}{q}\right) \sum_{r=0}^{q-1} Z_{\alpha}^{r} Z_{\alpha+1}^{-r}} \\
Z_{\alpha}=1 \otimes \ldots \otimes 1 \otimes Z \otimes 1 \otimes \ldots \otimes 1
\end{gathered}
$$

where $Z$ is a diagonal $q \times q$ matrix

$$
Z=\left(\begin{array}{cccc}
1 & 0 & \ldots & 0 \\
0 & w & \ldots & 0 \\
\vdots & \vdots & \ddots & \vdots \\
0 & 0 & \ldots & w^{q-1}
\end{array}\right) \quad, \quad Z^{q}=I_{q \times q} \quad, \quad w=e^{\frac{2 \pi i}{q}}
$$

The determinant of the transfer matrix can then be calculated using (22) and (24) (note that $\operatorname{Tr}(A \otimes B)=(\operatorname{Tr} A)(\operatorname{Tr} B)$ and $\operatorname{det}(\exp [\mathrm{A}])=\exp [\operatorname{Tr} \mathrm{A}])$

$$
\begin{aligned}
\operatorname{det} V_{1} & =\exp \left[\operatorname{Tr}\left(\frac{\tilde{k}}{2} \sum_{\alpha=1}^{n} X_{\alpha}\right)\right] \\
& =\exp \left[\frac{n \tilde{k}}{2} q^{n-1}(2-q)\right]
\end{aligned}
$$

and as $Z_{\alpha}^{r}$ is traceless for $r \neq 0$ (note that $\sum_{r=0}^{q-1} w^{(i-j) r}=q \delta_{i j}$ )

$$
\begin{aligned}
\operatorname{det} V_{2} & =\exp \left[\operatorname{Tr}\left(\frac{k}{q} \sum_{\alpha=1}^{n} \sum_{r=0}^{q-1} Z_{\alpha}^{r} Z_{\alpha+1}^{r}\right)\right] \\
& =\exp \left[n k q^{n-1}\right]
\end{aligned}
$$

and

$$
\operatorname{det}\left(V_{2} V_{1}^{\prime}\right)=\left(e^{k}-1\right)^{n q^{n}}\left(e^{k} e^{\tilde{k}}\right)^{n q^{n-1}}
$$


which has already been conjectured in [14]. It may be interesting to extend these results to lattices with different boundary conditions. It may also be useful to write a transfer matrix for the three dimensional Potts model and calculate its determinant. This decomposition of the transfer matrix may also be useful for obtaining other exact results for the two dimensional Potts model and maybe for calculation of its partition function which is still an unsolved problem.

\section{Conclusion}

In this work a conjectured formula by Chang and Shrock [14] for determinants of the transfer matrices of the q-state Potts model is proved.

\section{Acknowledgements}

We would like to thank R.J. Baxter for comments and suggestions. The Authors would like to thank the Isfahan University of Technology and Institute for Studies in Theoretical Physics and Mathematics for the financial support they made available to us.

\section{References}

[1] R. B. Potts, Proc. Camb. Phil. Soc. 48, 106 (1952).

[2] F. Y. Wu, Rev. Mod. Phys. 54 (1982) 235.

[3] S. Alexander, Phys. Lett. A54, 353 (1975).

[4] A. N. Berker, S. Oslund, and F.Putnam, Phys. Rev. B17, 3650 (1978).

[5] E. Domany et al, Phys. Rev. B18, 2209 (1978).

[6] R. J. Baxter, J. Phys. C 6 L445 (1973).

[7] R. J. Baxter et al, Proc. Roy. Soc. London, Ser. A 358, 535 (1978).

[8] R. J. Baxter, J. Stat. Phys. 28,1 (1982).

[9] R. J. Baxter, Proc. Roy. Soc. London, Ser. A 383, 43 (1982).

[10] M. P. M. den Nijis, J. Phys A 12, 1825 (1979); Phys. Rev. B27, 1674.

[11] J. L. Black and V. J. Emery, Phys. Rev. B23, 429 (1981).

[12] B. Nienhuis, J. Appl. Phys. 15, 199 (1982).

[13] V. S. Dotsenko, Nucl. Phys. B235, 54 (1984), and refs therein.

[14] S. Chang and R. Schrok, Physica A 296, 234-288 (2001). cond-mat/0011503. (e.g. Eq. 3.4.39 or 3.70 in Physica A )

[15] R. J. Baxter, Exactly Solved Models in St. Phys. (Academic Press, 1982).

[16] K. Huang, Statistical Physics, 2nd edition, (Wiley). Chapter 15.

[17] B. Bergersen and M. Plischke, Equilibrium Statistical Physics, 2nd edition, (World Scientific). Chapter 5.

[18] L. Onsager, Phys. Rev. 65, 117-49 (1944).

[19] B. Kaufmann, Phys. Rev. 76, 1232-1243 (1949).

[20] T. Shultz, D. Mattis and E. Lieb, Rev. Mod. Phys. 36, 856 (1964).

[21] P. Martin, Potts Models and related Problems in St. Phys. (World Scientific). 